\title{
Amoeboid Neutrophils with Few Granules in Childhood Acute Precursor B Cell Leukemia
}

\author{
Takeshi Asano', Kiyohiko Kaizu ${ }^{1}$ and Miho Maeda ${ }^{2}$ \\ ${ }^{1}$ Department of Pediatrics, Nippon Medical School Chiba Hokusoh Hospital \\ ${ }^{2}$ Department of Pediatrics, Nippon Medical School
}

\begin{abstract}
Background: We sometimes treat children with acute lymphoblastic leukemia in whom neutrophil function is impaired at diagnosis. Transmission electron microscopy enables more accurate assessment by providing greater morphological detail. Using transmission electron microscopy, we have found 2 types of neutrophils in the peripheral blood of children: 1) amoeboid neutrophils, which are characterized as amoeboid cells with pseudopodia and few granules, and 2) round neutrophils with many granules at different stages and glycogen particles.
\end{abstract}

Aim: To assess the pathological role of amoeboid neutrophils, we investigated amoeboid neutrophils in the peripheral blood of children with leukemia.

Methods: Amoeboid neutrophils were examined in peripheral blood from 12 children with acute B-cell precursor lymphoblastic leukemia (BCP-ALL). Eight children with short stature served as healthy control subjects.

Results: The percentage of amoeboid neutrophils (per total neutrophil count) at onset or relapse of BCP-ALL was significantly higher than at remission. Children with short stature showed a lower percentage of amoeboid neutrophils than did children with acute leukemia.

Conclusion: The presence of fewer intracellular granules in amoeboid neutrophils suggests lower neutrophil activity. These results indicate that amoeboid neutrophils in patients with BCP-ALL have lower function at onset and relapse.

(J Nippon Med Sch 2014; 81: 78-83)

Key words: amoeboid neutrophil, acute leukemia, childhood, electron microscopy

\section{Introduction}

Infection is a major obstacle for cancer chemotherapy. We have treated children with acute lymphoblastic leukemia (ALL) in whom severe infection developed at diagnosis despite neutrophil counts being in the normal range. These cases strongly suggest impaired neutrophil function in ALL at diagnosis.

Transmission electron microscopy is used to examine the characteristics of leukocytes ${ }^{1.2}$ and leukemic cells ${ }^{3}$ and enables more accurate assessment by providing morphological details not

Correspondence to Takeshi Asano, MD, Department of Pediatrics, Nippon Medical School Chiba Hokusoh Hospital, 1715 Kamagari, Inzai, Chiba 270-1694, Japan

E-mail: July1364@nms.ac.jp

Journal Website (http://www.nms.ac.jp/jnms/) 
discernible with light microscopy. During the routine ultrastructural examination of peripheral blood from children with acute leukemias at our institution, we have often noted amoeboid neutrophils with few granules which differed from round neutrophils with many granules at different stages (azurophilc, specific, and secretory granules) ${ }^{4}$. However, the physiological and pathological roles of these amoeboid neutrophils are unclear, particularly in childhood acute leukemia. In this context, amoeboid neutrophils in B-cell precursor ALL (BCP-ALL) were analyzed at onset or relapse and at remission.

\section{Materials and Methods}

\section{Patients and Controls}

Peripheral blood samples remaining after ordinary clinical use were used for this study after informed consent had been obtained from patients or guardians. Twelve patients, aged 1 to 17 years (average age, 9.7 \pm 5.4 years), with BCP-ALL were enrolled in this study. Four children had relapsed disease, and the others had newly diagnosed disease. Two patients provided samples at both onset and relapse. The diagnosis of BCP-ALL was confirmed with standard methods. All patients were treated with the Tokyo Children's Cancer Study Group ALL protocol $^{5,6}$. Although some children had infectious disease on admission, the pathogen could not be identified in any of them. For ethical reasons, obtaining blood samples from healthy children for research is extremely difficult in Japan. In addition, peripheral blood from patients with infectious diseases or inflammatory diseases could not be used as specimens from healthy control subjects for assessing neutrophil function. Therefore, we examined the percentages of amoeboid neutrophils in blood samples from 8 children with short stature (average age, 9.9 \pm 1.3 years), who we assumed to have normal neutrophil function, as control specimens from subjects without infectious, inflammatory, or malignant conditions.

\section{Definition of Amoeboid Neutrophils}

Amoeboid neutrophils are amoeboid cells ranging from 13 to $18 \mu \mathrm{m}$ in greatest diameter containing few granules and glycogen particles and having pseudopodia with no granules (Fig. 1). In contrast, round neutrophils with many granules at different stages (azulophilc, specific, and secretory granules) ${ }^{4}$ are seen in the peripheral blood (Fig. 1).

\section{Microscopic Procedure}

For electron microscopic observation, sample preparation was as follows ${ }^{7}$. After centrifugation of heparinized blood samples, plasma was replaced with a $2.5 \%$ glutaraldehyde in phosphate buffer, $\mathrm{pH}$ 7.4, for fixation. After hardening, the buffy coat disk was removed and minced into small pieces according to a previously reported method ${ }^{5}$. Samples were postfixed in $1 \%$ osmium tetroxide, dehydrated in a graded alcohol series, and embedded in epoxy resin (Epok 812, Okenshoji Co. Ltd., Tokyo, Japan). Ultrathin sections were cut on an ultramicrotome, stained with uranyl acetate and lead citrate, and examined under an electron microscope at $80 \mathrm{kV}$ (Hitachi 7500, Hitachi Co., Ltd., Ibaraki, Japan). The buffy coat disk included thrombocytes on one side and red blood cells on the other side to overcome any tendency for differential layering of the various types of leukocytes. Leukocytes were counted in at least 12 electron micrographs randomly taken from each specimen. The percentages of total neutrophils and amoeboid neutrophils were determined in the approximately 200 leukocytes counted in each specimen. Subsequently, the percentage of amoeboid neutrophils per total leukocytes was calculated in each case.

\section{Statistical Analysis}

Statistical analysis was performed with the Kruskal-Wallis H-test with Bonferroni correction and, if significant, a Mann-Whitney $U$-test. Comparisons of the percentages of amoeboid neutrophils at onset or relapse and in remission in the same patients were performed with paired Wilcoxon analysis.

\section{Results}

Ultrastructural observation of peripheral blood cells showed 5 major, distinct morphologic types of leukocytes: lymphocytes, neutrophils, monocytes, 


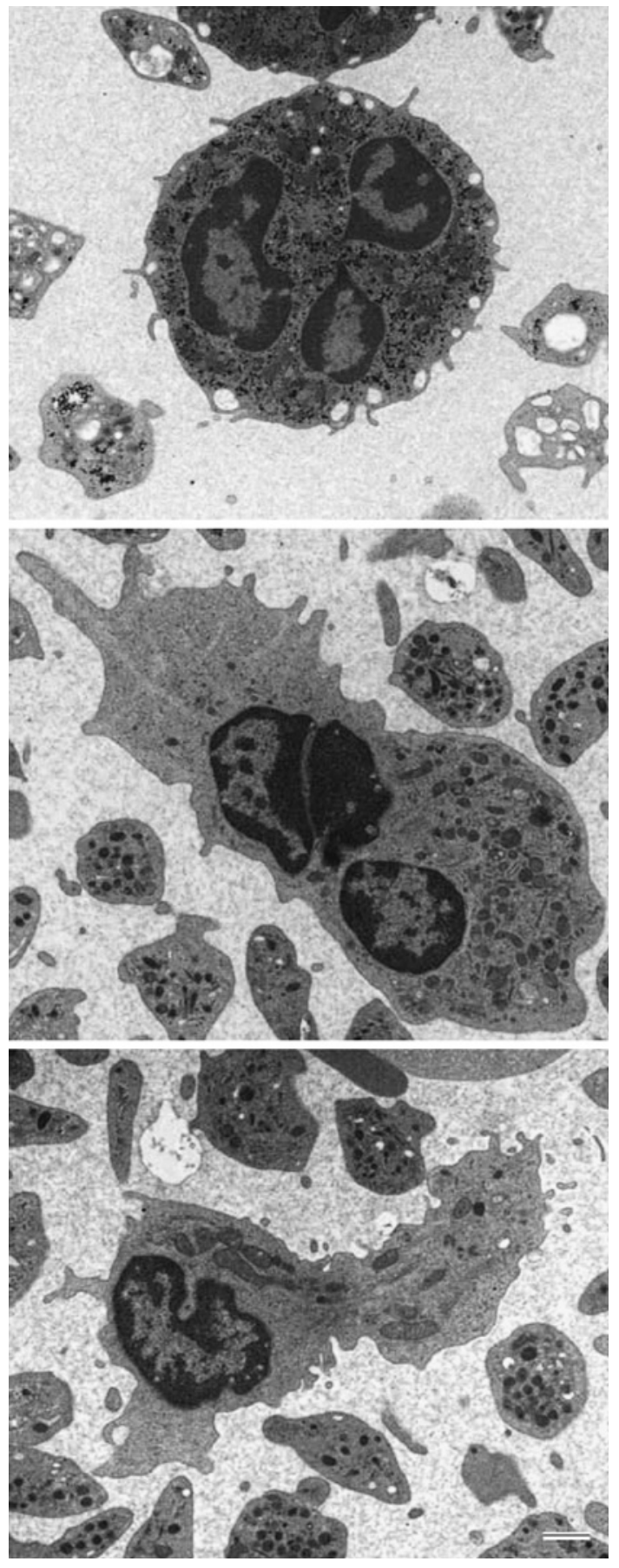

Fig. 1 Representative electron micrographs showing features of amoeboid neutrophils with few granules and of round neutrophils Amoeboid neutrophils (bottom and middle pictures) are amoeboid cells ranging from 13 to $18 \mu \mathrm{m}$ with pseudopodia and have fewer granules and glycogen particles than do round neutrophils, which have many granules at different stages (azulophilc, specific, and secretory granules) (top picture). The bottom picture shows large pseudopodia and degranulation. In addition, no granules are observed in the pseudopodia of amoeboid neutrophils.

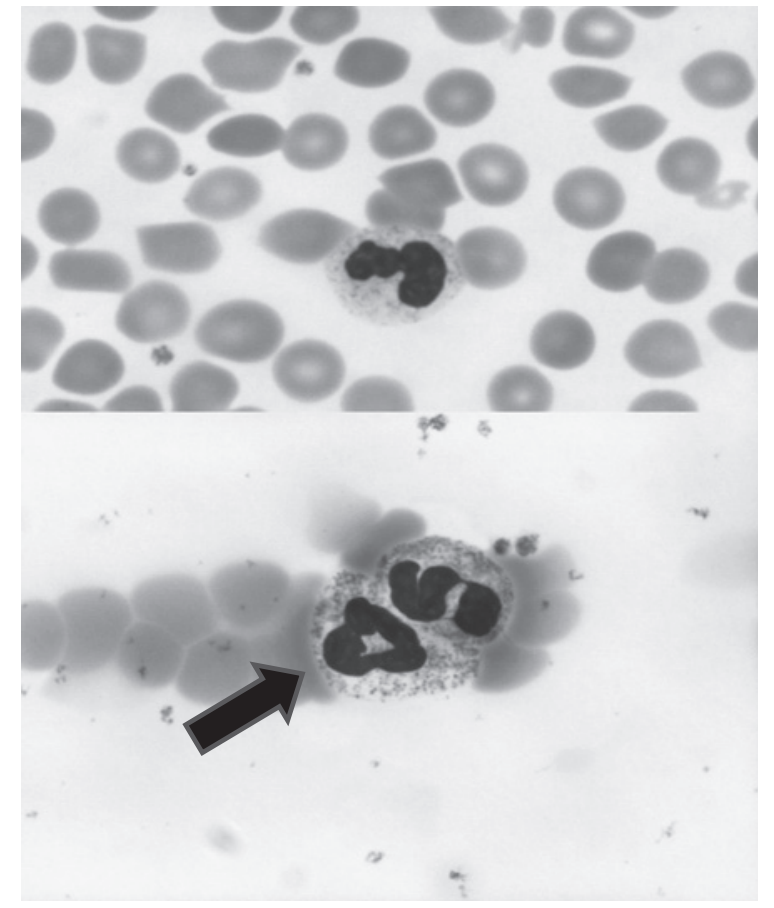

Fig. 2 Light microscopic findings of amoeboid-like cells and round-like cells from smear specimens from a patient with ALL in remission

A neutrophil in the upper window showed a round shape and fine granules suggesting a round neutrophil. A neutrophil in the lower window (arrow) showed an amoeboid-like shape and coarse granules suggesting an amoeboid neutrophil. However, these differences were not clear

eosinophils, and basophils. Round neutrophils were observed in the red blood cell side of the buffy coat disk. The cytoplasm of round neutrophils contained many glycogen particles and various granules, including azurophilic granules and specific granules, and secretory vesicles (Fig. 1).

On the other hand, amoeboid neutrophils were usually seen on the thrombocyte side of the buffy coat. Amoeboid neutrophils are amoeboid cells ranging from 13 to $18 \mu \mathrm{m}$ at their greatest diameter and exhibit pseudopodia. Amoeboid neutrophils are sometimes found together with thrombocytes. The amoeboid neutrophils often showed low numbers of granules and glycogen particles. In addition, no granules were observed in the pseudopodia of amoeboid neutrophils (Fig. 1).

Although we tried to distinguish amoeboid cells from round cells by means of light microscopy, doing 


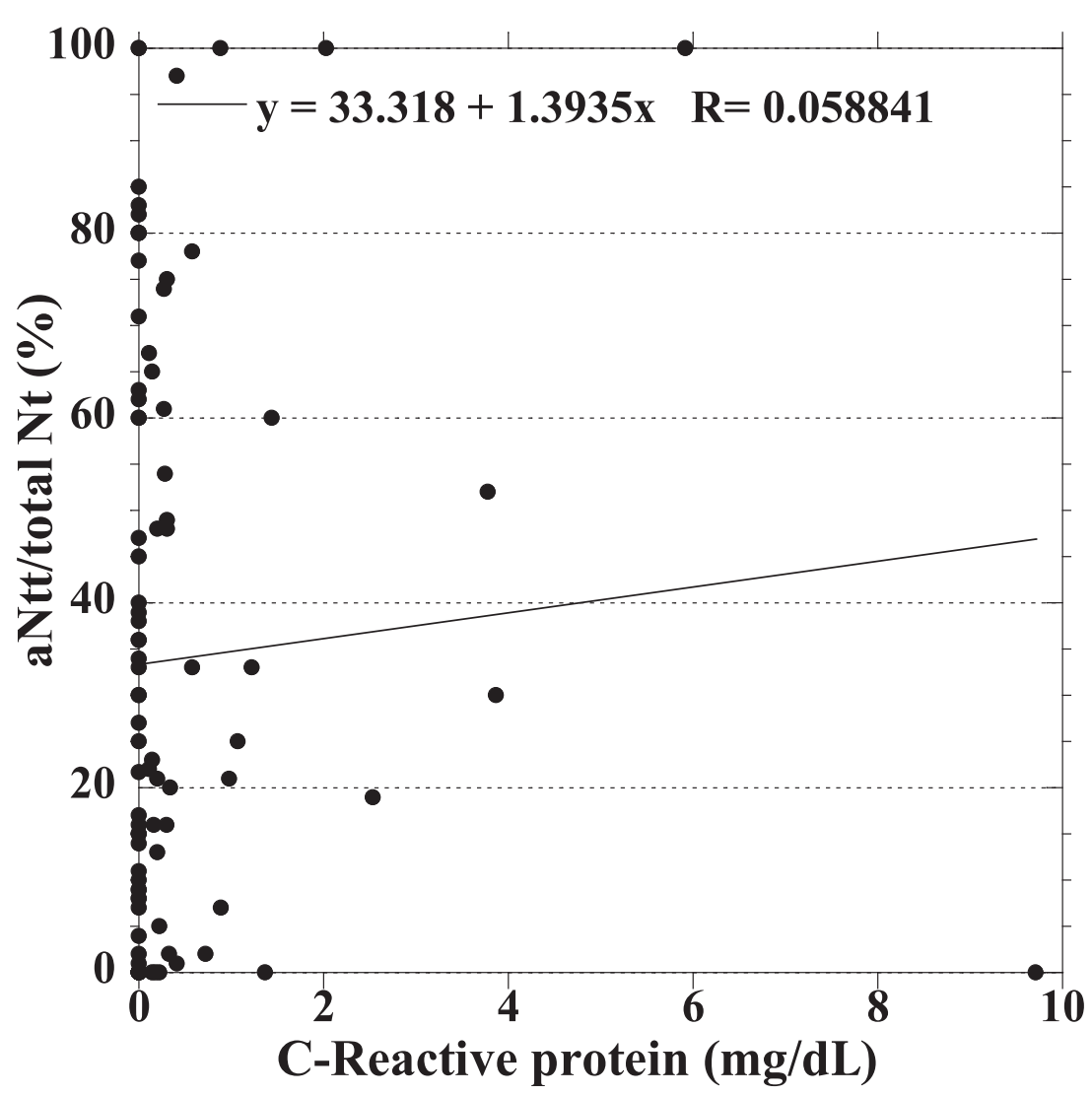

Fig. 3 Relation between an increased percentage of amoeboid neutrophils and the C-reactive protein value

There was no statistically significant relationship between the percentage of amoeboid neutrophils and the C-reactive protein value.

so was not possible with smear specimens and clot section specimens of peripheral blood (Fig. 2).

\section{Percentage of Amoeboid Neutrophils}

The percentage of amoeboid neutrophils (per total neutrophil count) was significantly higher at onset or relapse of $\mathrm{BCP}-\mathrm{ALL}(43.3 \% \pm 34.9 \%, 2$ of 12 cases $)$ than in remission $(28.1 \% \pm 27.0 \%, \mathrm{p}=0.005)$, according to paired Wilcoxon analysis. However, we could find no relation between the percentage or count of amoeboid neutrophils and the severity of infectious events, represented by the C-reactive protein value (Fig. 3). The percentage of amoeboid neutrophils was significantly higher in BCP-ALL at onset or relapse than in control subjects (Fig. 4).

\section{Discussion}

Several studies of neutrophil function in patients with $\mathrm{ALL}^{8-11}$ have used chemotaxis assay, $\mathrm{H}_{2} \mathrm{O}_{2}$ and
$\mathrm{O}_{2}$ production, bactericidal activity, oxidative burst, and phagocytic activity to evaluate neutrophil function. These studies have shown impaired chemotaxis $^{7}$, phagocytic index and local leukocyte mobilization $^{8}$, and oxidative burst ${ }^{11}$, at the onset of ALL. Some impaired functions recovered to normal levels after treatment ${ }^{8,911}$. These studies have indicated that some bactericidal functions of neutrophils are impaired at the onset of childhood ALL but are restored during remission. The present study has found that novel morphological findings might be related to neutrophil dysfunction in children with ALL.

The amoeboid neutrophils observed with electron microscopy in the present study had fewer granules and glycogen particles and had pseudopodia. Their large size suggests that these amoeboid neutrophils had undergone frustrated phagocytosis ${ }^{12,13}$, with few granules remaining ${ }^{14,15}$. Fewer intracellular glycogen granules, which are the main energy source for 


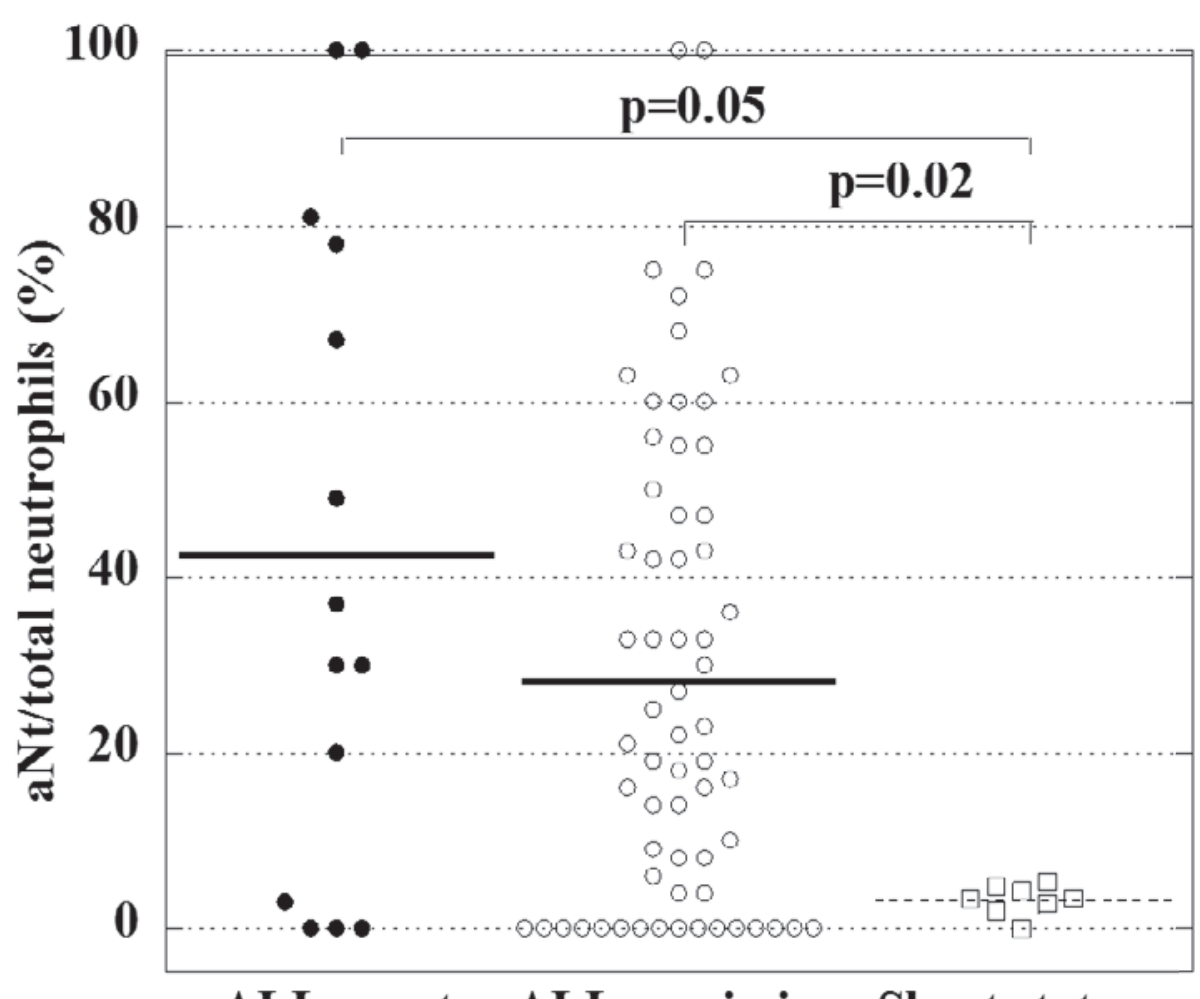

ALL onset ALL remission Short stature

Fig. 4 Percentages of amoeboid neutrophils in samples of peripheral blood from patients with BCP-ALL at onset or relapse and in remission and from control subjects

The percentage of amoeboid neutrophils was calculated as the number of amoeboid neutrophils divided by total number of neutrophils on electron microscopy. The Mann-Whitney $U$-test was used to compare the percentage of amoeboid neutrophils in children with BCP-ALL at onset or relapse and in remission with that in children with short stature as a control.

BCP-ALL: B cell precursor acute lymphoblastic leukemia

neutrophils, strongly indicate that amoeboid neutrophils have impaired energy supply, leading to impaired immunological function ${ }^{16}$. Thus, the amoeboid neutrophils we observed at onset of ALL may have been neutrophils exhausted after frustrated phagocytosis.

We attempted to distinguish between 2 types of granulocytes with light microscopy. However granulocytes from patients with ALL could not be clearly distinguished with light microscopy (Fig. 2). Thus, we believe that electron microscopic analysis in this study might be a novel method for identifying amoeboid neutrophils.

In conclusion, we have reported an increased percentage of amoeboid neutrophils at onset or relapse in patients with childhood BCP-ALL. Children with BCP-ALL and suppressed neutrophil function before treatment are predisposed to severe infections after chemotherapy. The present results suggest a method for estimating the risk of infection in patients receiving high-dose chemotherapy.

Acknowledgement: We are grateful to Ms. Akiko Adachi, Mr. Shigeru Sato, Mr. Yoshihiro Sasaki and Mr. Oguro for preparing the samples for the electron microscopic study.

Conflict of interest: The authors declare no competing financial interests.

\section{References}

1. Ryan DH: Examination of the blood. In Williams Hematology (Wonsiewicz M, Edmonson KG, eds), Sixth edition. 2001; pp 9-16, The McGraw-Hill.

2. Bainton DF: Morphology of neutrophils, eosinophils 
and basophils. In Williams Hematology (Wonsiewicz M, Edmonson KG, eds), Sixth edition. 2001; pp 729733, The McGraw-Hill.

3. Dickerson GR: Leukemia. In Diagnostic Electron Microscopy. A text/Atlas, Second edition. 1998; pp 217-246, Springer-Verlag, New York.

4. Adachi A, Sato S, Sasaki Y, et al.: Electron microscopic studies on the occurrence of activated neutrohils in peripheral blood of children with acute leukemias. J Submicrosc Cyto Pathol 2005; 37: 13-18.

5. Manabe A, Ohara A, Hasegawa D, Koh K, Saito T, Kiyokawa N: Significance of the complete clearance of peripheral blasts after 7 days of prednisolone treatment in children with acute lymphoblastic leukemia: the Tokyo Children's Cancer Study Group Study L99-15. Haematologica 2008; 93: 1155-1160.

6. Narazaki H, Kaizu K, Miyatake C, Koizumi S, Asano T, Fujino O: Delayed-type Hypersensitivity in Response to L-asparaginase in a Case of Acute Lymphoblastic Leukemia. J Nippon Med Sch 2012; 79: 489-493.

7. Douglas RA: A method of preparing peripheral leucocytes for electron microscopy. J Ultrastr Res 1965; 13: 263-268.

8. Al-Nakeeb S, Thompson EN: Neutrophil chemotaxis and random migration in acute lymphoblastic leukaemia of childhood. Cellular and humoral aspects. Arch Dis Child 1980; 55: 299-303.

9. Hofmann WK, Stauch M, Hoffken K: Impaired granulocytic function in patients with acute leukemia: only partial normalization after successful remission-inducing treatment. J Cancer Res Clin Oncol 1998; 124: 113-116.

10. Lejeune M, Ferster A, Cantinieaux B, Sariban E:
Prolonged but reversible neutrophil dysfunctions differently sensitive to G-CSF in children with ALL. Br J Hematol 1998; 102: 1284-1291.

11. Tanaka F, Goto H, Yokosuka T, et al:: Suppressed neutrophil function in children with acute lymphoblastic leukemia. Int J Hematol 2009; 90: 311317.

12. Borregaard N: The human neutrophil. Function and dysfunction. Eur J Haematol 1988; 41: 401-413.

13. Kruskal BA, Maxfield FR: Cytosolic free calcium increases before and oscillates during frustrated phagocytosis in macrophages. J Cell Biol 1987; 105: 2685-2693.

14. Henson PM: The immunological release of constituents from neutrophil leukocytes. 1. The role of antibody and complement on nonphagocytosable surface or phagocytosable particles. J Immunol 1971; 107: 1535-1546.

15. Marmon AM, Zucker-Franklin D: Neutrophils. In atlas of Blood Cells; Function and Pathology (ZuckerFranklin D, Greaves MF, Grossi CE, Marmont AM, eds), Second edition. 1988; pp 170-185, edi-ermes Milano.

16. Cohn ZA, Morse SI: Functional and metabolic properties of polymorphonuclear leucocytes. I. Observations on the requirements and consequences of particle ingestion. J Exp Med 1960; 111: 667-687.

(Received, July 10, 2013) (Accepted, August 13, 2013) 